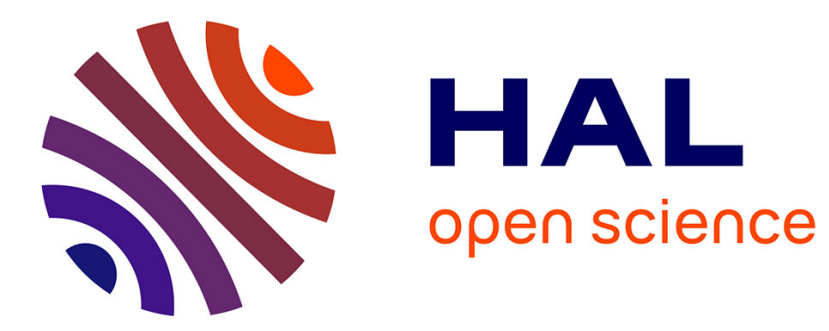

\title{
Distance estimations in unknown sea underwater conditions by power LED for robotics swarms
}

Ramiro Dell'Erba

\section{To cite this version:}

Ramiro Dell'Erba. Distance estimations in unknown sea underwater conditions by power LED for robotics swarms. Continuum Mechanics and Thermodynamics, 2021, 10.1007/s00161-020-00889-x . hal-03119198

\author{
HAL Id: hal-03119198 \\ https://hal.science/hal-03119198
}

Submitted on 23 Jan 2021

HAL is a multi-disciplinary open access archive for the deposit and dissemination of scientific research documents, whether they are published or not. The documents may come from teaching and research institutions in France or abroad, or from public or private research centers.
L'archive ouverte pluridisciplinaire HAL, est destinée au dépôt et à la diffusion de documents scientifiques de niveau recherche, publiés ou non, émanant des établissements d'enseignement et de recherche français ou étrangers, des laboratoires publics ou privés. 


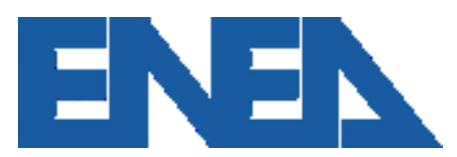

Italian National Agency for New Technologies, Energy and Sustainable Economic Development http://www.enea.it/en http://robotica.casaccia.enea.it/index.php?lang=en

This paper is a pre-print. The final paper is available on: dell'Erba, R. "Distance estimations in unknown sea underwater conditions by power LED for robotics swarms". Continuum Mech. Thermodyn. 33, 97-106 (2021). https://doi.org/10.1007/s00161-02000889-x 


\title{
Distance estimations in unknown sea underwater conditions by power LED for robotics swarms
}

Ramiro dell'Erba

ENEA Technical Unit technologies for energy and industry - Robotics Laboratory

ramiro.dellerba@enea.it

ORCID id 0000-0002-4857-4025

\begin{abstract}
The aim of this paper is to measure distances between autonomous underwater vehicles using power LED as light source and photodiode as receiver in unknown light water adsorption conditions. The method is based on the attenuation of the light signal, depending principally on distance and water characteristics. In a previous paper we proposed the use of a cheap power LED system to support acoustic devices in localization and configuration computation of an underwater robotics swarm. The idea was based on the exchange of light signals of different wavelengths, to reduce the noise, generated by LEDs; the unknown water conditions, affecting the light propagation, required a local measure of the absorption function $a(\lambda)$ to obtain the distance between the source and the receiver. To avoid the use of many different wavelength LEDs we investigated the influence of power and flash duration on the LED spectral emission. Starting from this experience we are now able to measure distances without the unpractical local measure of the absorption function. In this paper we show as, modifying frequency, we shall be able to measure $a(\lambda)$ and, consequently, the distance $d$.
\end{abstract}

\section{Keywords}

LED, Autonomous vehicle, underwater robot, distances measurement.

\section{Introduction}

ENEA is working in robotics since a long time (1961) and underwater robotics is a key topic of our laboratory [1]-[3]. Some years ago, (2006) we moved our attention from single autonomous underwater vehicle (AUV) to a swarm of very low cost cooperating robots. The use of underwater autonomous vessels or rovers has been proven to be a powerful tool in many submarine tasks for monitoring, exploration, search, rescue and many other applications but it still is expensive, because it requires the support of an equipped ship. To make AUV competitive with rovers many problems are still to be solved, especially about power availability, information processing, navigation, and control [4], [5], [6].

Performances improvement of AUV can be achieved using of a large number of low cost cooperating mini AUV, [7]. In fact a swarm is able to perform tasks in a more fast and robust way with respect of a single machine. Perhaps the most important feature of a swarm is its capability to span from the surface to the basement; this allows ensuring a quasi-real time communication with the human operator and, therefore, to interact with the underwater system using a remote console that can be locate also on the coast. The swarm allow realizing a multi-hop submarine network where the nodes of the net are the elements of the swarm Therefore we are dealing with a variable geometry network. This could limit the use of the expensive surface ships to the deployment phase, taking advantage of the parallel exploration to shorten times and have many other advantages [5], [8]. As example other advantages, using a swarm approach, lies in the speed-up coming from the parallelism and in the increase of reliability by redundancy [9]; the lack of one member can be easily managed by redistributing the job among the others like, in natural systems, for the bees [10]. Last, but not least, a swarm can interact with a human operator as a single object, without the problem of controlling a large number of individuals, once a swarm control layer it has been developed.

The concept of robot swarms is a study theme by the scientific community since several years. The realization of swarms using a different numbers of cooperating robots has been successfully tried [11], [12], [13], but in underwater environment it is still a challenge [14], [15], [16].

One of the most important tasks for an underwater vehicle is its localization into the sea; a swarm has the supplementary task to know its geometric configuration, which means to be aware of the relative position of some other robots, typically the neighbours is enough. To get absolute localization of the whole swarm at least one element of the swarm must have a 
precise localization. Therefore one element comes out on the surface to fix its position by GPS; that of the other swarm elements are computed from the knowledge of the relative position between them. The knowledge of the geometric configuration is a very important issue for many applications; it often is depending by the assigned task that can also be changed during the mission [17]. One of the reasons to vary the geometric configuration is to have the most efficient perception (in general meaning, vision, acoustical data, magnetic measurements) of the surrounding environment; but there are also needs connected with the optimization of internal and external channel communication network. This knowledge was, in a first release of underwater network, achieved using the time-of-flight of a dedicated acoustic modem to obtain the distance among the first neighbours. Unfortunately it is well known as reflections, fading and other phenomena make difficult and sometimes not reliable measurements that are involving acoustic devices. The need to continuously exchange data among the nodes, to get the distances, represents a considerable burden for the network operation. In fact to calculate the network configuration, also using suitable algorithms [1], forces to frequent short messages that strongly deoptimize the exploitation of the communication channel, mainly for the long times needed to switch from a message to another one. A possible solution, which improves both the time allocation, in the acoustical protocols, and unloads the acoustical channel burden, is to couple the acoustic protocol with an optical device. The intention is to collect distances measurements between the robots more precise, using sensor data fusion.

Optical methods are very powerful but their performances are strongly affected by many variable parameters like salinity, turbidity, the presence of dissolved substances that change the colour and the transparency of the water in all the optical bands. Moreover the presence of solar radiation, in shallow water, heavily affects the signal to noise ratio. Our current approach uses a mixed strategy based on the variable exploitation of the optical channel depending on the environmental conditions. In favourable conditions the transmission protocol will freely decide which channel to adopt depending on the priority, i.e. distance-to-cover and dimension of the message itself. In less favourable conditions the optical channel will be limited to the fundamental synchronization task, generating a light lamp that will optimize the message passing through the optical channel. Any way backup solutions, based on the "all acoustical" approach, must remain available because there is the possibility to meet dirty waters with no practical possibility to use light signals.

It must be outlined that only dense swarms can take advantage of such approach because only in these situations, with internal distances ranging from few meters to a maximum of 20 meters, there are the conditions suitable to use light signals for sync and measurements. A further extension of the problem presented here can be performed by considering the interplay between swarm robotics and newly conceived materials, the so-called metamaterials. Some examples of these materials can be found in [18], [19], [20], [21], [22].

\section{State of the art}

In a previous paper [23] we have considered cheap power LED to build an optical modem and tried to stress power supply and flash duration to vary light emission characteristics; examples of typical emission are shown in Figure 1 and Figure 2. Note that the white light is obtained by fluorescence of coating using a blue LED, as can be deducted by the presence of a double peak in the plot. We are strongly interested in the variation of the emission peak with the power supply; the reason is economical. Because we are thinking in terms of a swarm if we use a complex variable frequency LED the cost can increase rapidly owing to the large element number of the swarm. Therefore, we want to be able to modify, in a little range, the frequency of the peak emission by the power tension, using a cheap LED and use this variation for our aims. The peak emission vs. power supply has been measured and the results are shown in Figure 3 for the white LED and in Figure 4 for the blue LED. The work has been performed by instrument with a resolution of $0.5 \mathrm{~nm}$ wavelength. We did this experimental job because the LEDs technical specifications from data sheet were referred to the static features of electric power and luminance so we had the need to carry on a more precise characterization of these diodes to study their applicability in signaling and communication.

Because the water adsorption is generally unknown in the old paper we performed a local measurement of the adsorption coefficient on the known head-tale distance, and use it to compute the unknown distances between the robots. This implies we have a LED emission on the head of our torpedo like robot and a photodiode on the tale. This determines constructive problems of the optical modem so far, we studied to got rid of this architecture and we are now able to measure AUVs distance just performing more measurements in different conditions instead of the head-tale measurement. So far, we stress emission to get information useful to model water attenuation coefficient and, consequently, the distance. 


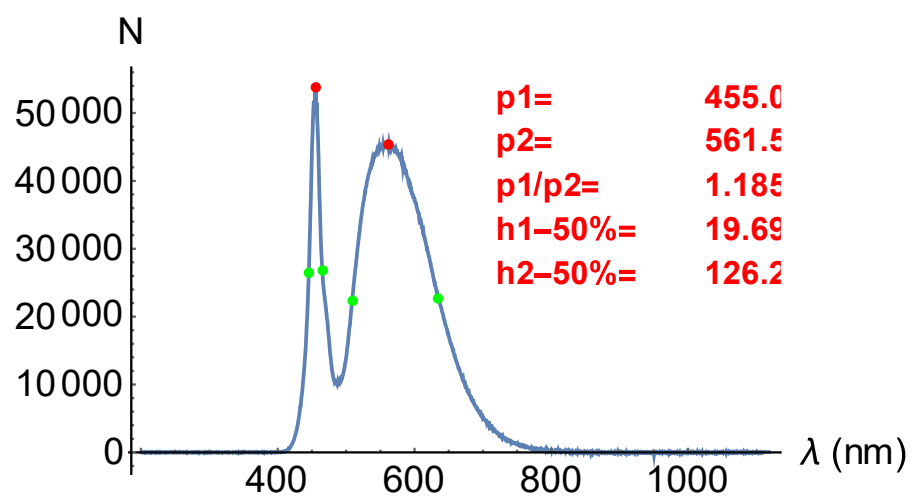

Figure 1 White LED typical emission plot

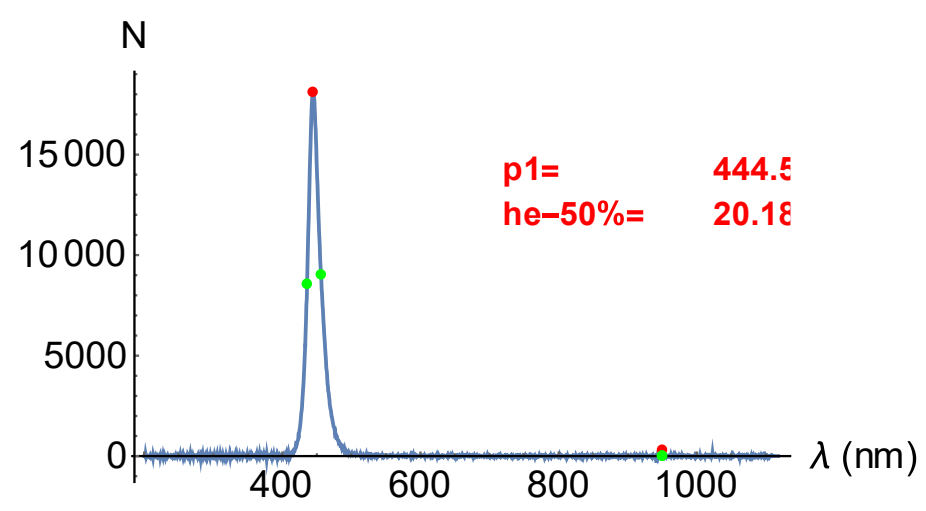

Figure 2 Blue LED typical emission plot.

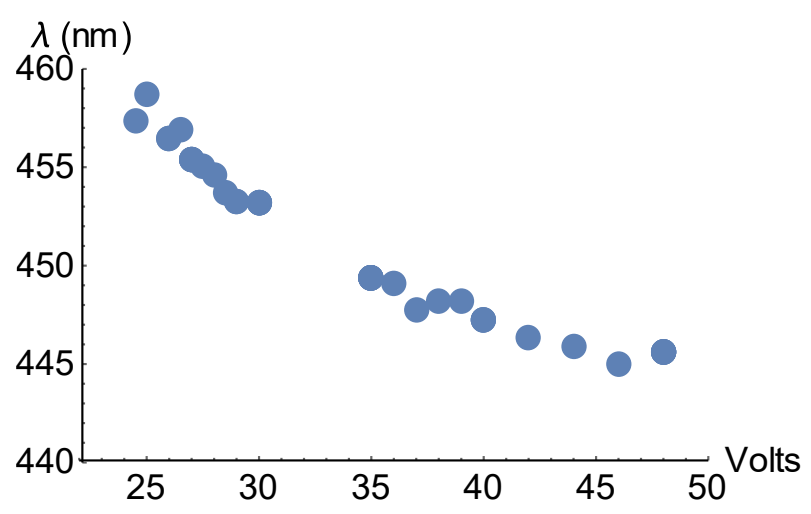

Figure 3 White LED first peak shift value vs. power tension.

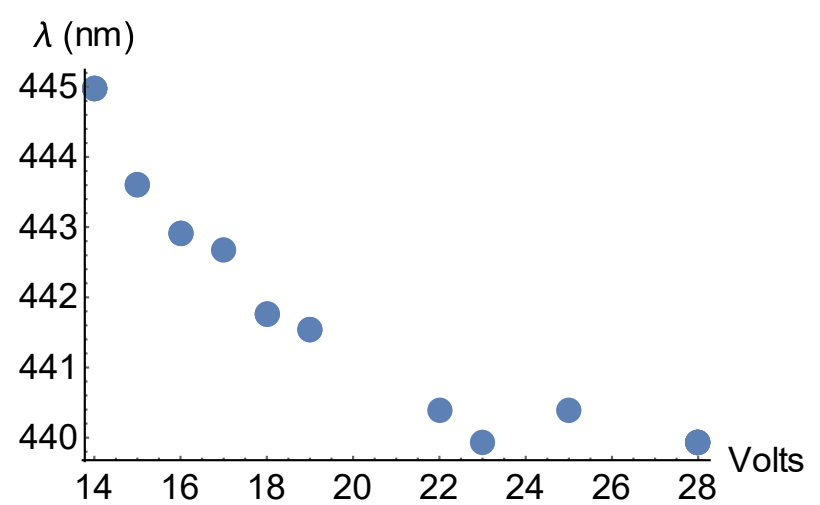

Figure 4 Blue LED peak shift vs. power tension. 


\section{Our prototype}

In Figure 5 and Figure 6 the optical modem prototype and the robot swarm element, realized in our laboratory, are shown. Robot's characteristics are the following: Max depth $100 \mathrm{~m}$; Max speed $4 \mathrm{~km} / \mathrm{h}$; Weight about $20 \mathrm{~kg}$; Autonomy 3hrs; Dimensions are $1.20 \mathrm{~m} \mathrm{X} 0.20 \mathrm{~m}$ diameter. Standard sensors include a stereoscopic camera, sonar, accelerometer, compass, depth meter. Remember that we are dealing with a system thought as to be a component of a swarm of about 20 objects, so the distances between robots are between 3 and $50 \mathrm{~m}$. Therefore, the maximum distance possible between two robots is about $1000 \mathrm{~m}$, as a very particular alignment case; the average value of the distances of the neighbors is considered about $10 \mathrm{~m}$. In underwater world a severe limitation to our communications technology is, perhaps, the main drawback: the physical medium only permits acoustical channels, since electromagnetic waves are rapidly damped [24]. The acoustical technology has limited performances; the band pass increase with the frequency but the signal is more rapidly damped, limiting useful range [25]. Technology of dense swarms is an answer to these problems with larger acoustical bands and allowing other physical channels, like optical, to be exploited. This paper explores the opportunities offered by simple and economical optical sources to perform swarm localization and communication.

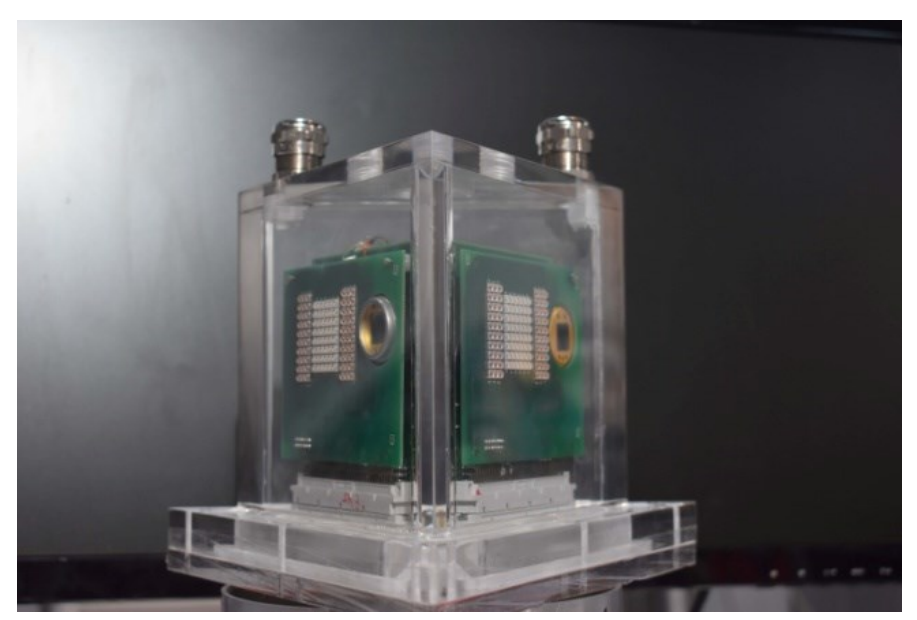

Figure 5 Optical modem. Square white Led, rectangular blue LED and circular photodiode are visible. To cover $360^{\circ}$ three systems are implemented.

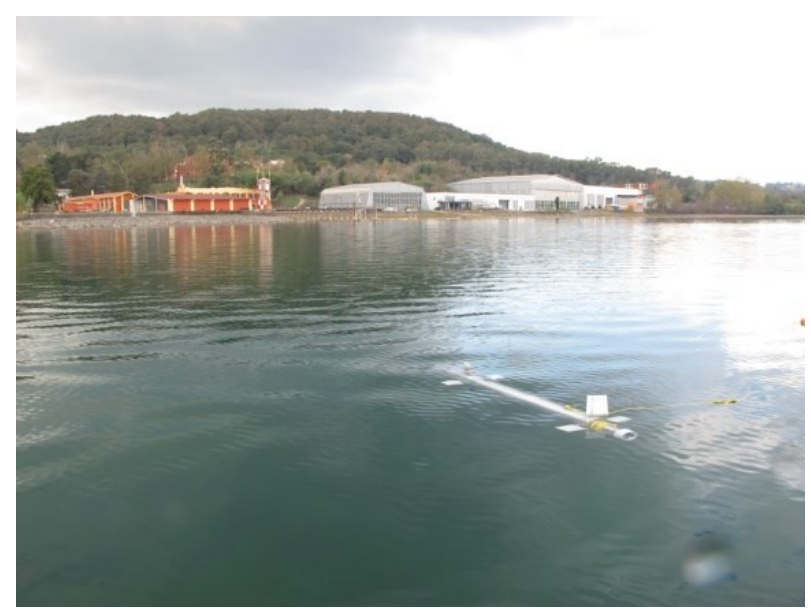

Figure 6 Robot prototype during the test in Bracciano's lake. 


\section{Theoretical approach to the problem}

Transmission of an electromagnetic wave through a material is governed by its electric and magnetic properties as described by Maxwell's equations. The response of a material to an electromagnetic field is described by the frequencydependent dielectric and magnetic tensorial parameters. Light propagation in a medium induces a medium response because of interaction of light with electrons and nuclei in the medium. The medium response to this interaction affecting the light propagation and generating various optical effects. This response, in the case of a sufficiently weak field can it be regarded as linear.

Light absorption by a medium, as a function of distance from the source and the wavelength, is a well-known phenomenon. In a first approximation, for a planar wave, like in case of laser source, we can assume an exponential law for the decay of the signal intensity.

$$
\mathrm{I}=\mathrm{I}_{0} \mathrm{e}^{-\mathrm{a}(\lambda) \mathrm{d}}
$$

Where $I$ is the measured intensity signal at the distance $d, I_{0}$ the emitted intensity, $a(\lambda)$ the absorption function typical of the medium.

In the case of spherical wave, owing to the energy balance

$$
\mathrm{I}=\frac{\mathrm{I}_{0} \mathrm{e}^{-\mathrm{a}(\lambda) \mathrm{d}}}{\mathrm{d}^{2}}
$$

Where $I$ is the intensity measured on the receiving surface unit. Typically, you have to consider a more complicate shape emission diagram for the LED diode described by a shape function $f(\vartheta, \varphi)$ in front of the fraction; in our case the LED have an emission of $60^{\circ} \times 60^{\circ}$ therefore the energy is spread on this solid angle. The $a(\lambda)$, describing how the signal is attenuated as function of the wave length lambda, is strongly affected by the water conditions as can be seen in Figure 7 whose data are due to the courtesy of [26]; it is possible to see as, in clear water that means lower $a(\lambda)$ value, this function has a minimum and high visible frequency are adsorbed less than lower. For some useful results in the field of wave propagation, also in generalized media, one can refer to the following papers [27], [28], [29], [30], [31], [32], [33].

The measured intensity, owing to the spread of the LED emission, is the integral, over all the wavelengths of the equations 1 and 2 . We are assuming that water characteristics do not vary in the volume containing the robots. The problem we are facing is how to measure the absorption function and the distance by our measurements. To this aim we distinguish two cases.

In the first case the wave can be considered planar and the measurements are performed in a short time range so we can consider fixed the robot position; we only stress the diode emission.

In the second case the wave is spherical and we can move the robots during the measurements.

Working on Eq.1 and Eq.2 we can note that in planar wave case to move the robots in two different and unknown position only allow us to obtain the ratio $d_{1} / d_{2}$ but give not information on the $a(\lambda)$ function. In the same way we are not able to obtain separate information on $a(\lambda)$ and $d$ in spherical wave case without the device's displacements.

Before starting we try to have an idea about the orders of magnitude of the attenuation in our case of interest. In we consider a distance of $10 \mathrm{~m}$, a radius of receiving photodiode of $0,02 \mathrm{~m}$ and the case of clear water $\left(a(\lambda)\right.$ about $\left.0.02 \mathrm{~m}^{-1}\right)$ we get a signal attenuation of $10 \%$; for the planar wave and $20 \%$ for the spherical one. We have considered $1 / 3$ as shape factor for the spherical wave because the Led emission diode is not omnidirectional. In case of turbid water $\left(a(\lambda)\right.$ about $\left.0.2 \mathrm{~m}^{-1}\right)$ the attenuation was about $60 \%$ and about $90 \%$ in planar and spherical wave case respectively. 


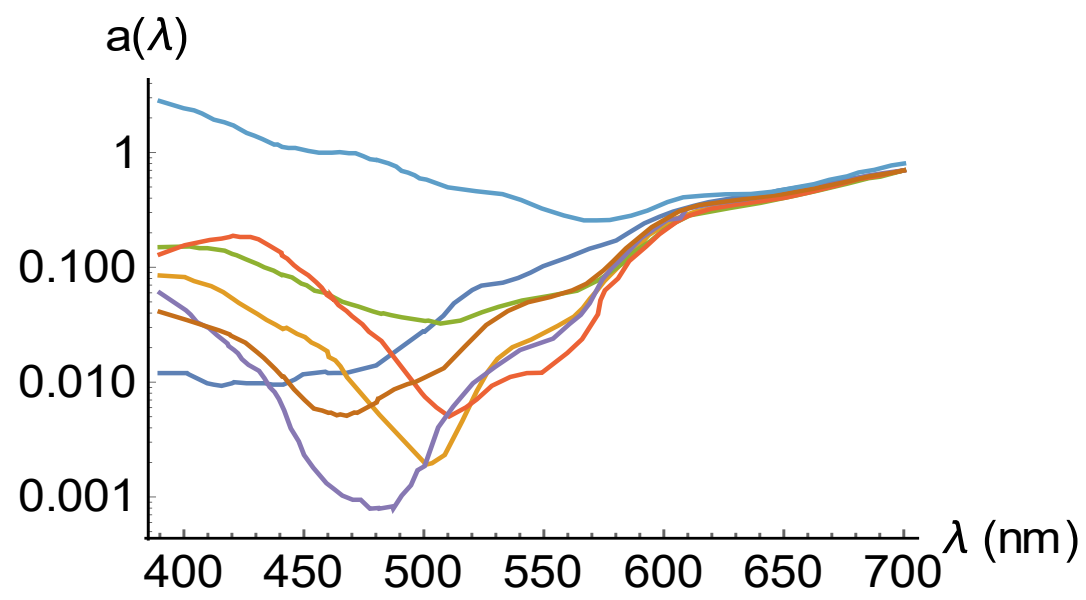

Figure 7 Light absorption in sea water in different condition [26].

Consider now the case where distance between the robots cannot be varied and we are working with a planar wave. There is no way to obtain, $a(\lambda)$ and $d$ separately by a simple measure if the intensity $I$ or working on a measurement set. This because the two objects are linked by multiplicative way and the exponential function. We always get the product. Anyway, stressing power, we can measure its derivative with frequency.

$$
\frac{\partial I}{\partial \lambda}=-a^{\prime}(\lambda) d I_{0} e^{-a(\lambda) d}
$$

Where $\delta \lambda$ is referring to the peak shift of the LED as measured in Figure 3 and Figure 4. Dividing by the intensity, $I$, and multiply the $\log$ of the intensity we can obtain the differential equation

$$
\frac{a^{\prime}(\lambda)}{a(\lambda)}=\frac{1}{I \log \left(I / I_{0}\right)} \frac{\partial I}{\partial \lambda}
$$

And in the same way

$$
\frac{a\left(\lambda_{1}\right)}{a\left(\lambda_{2}\right)}=\frac{\log \left(I\left(\lambda_{1}\right) / I_{0}\right)}{\log \left(I\left(\lambda_{2}\right) / I_{0}\right)}
$$

The ratio is available for any couple of frequency we are able to generate.

The differential Equation (Eq.4) could be integrated but we do not know any Cauchy condition a( $\left.\lambda_{0}\right)$ and the ratio (Eq.5) cannot be useful to suggest it. The problem is stiff so we are still no able to separate and obtain the parameters of the equation. If we consider the frequency range between $440 \mathrm{~nm}$ and $460 \mathrm{~nm}$, where we are able to operate, we can ask the following question: Does exist a simple model, depending by parameters, able to fit any water conditions $a(\lambda)$ (as showed in Figure 7) in that range? And if it exists, are we able, with our measurements, to obtain the parameters? The answer is yes but only if the model keeps separate the two parameters by not multiplicative operation. So far, the idea is to find a simple model (avoiding the multiplicative presence of $a(\lambda)$ and $d$ ) to characterize water, estimate its parameters by our measurements and obtain $a(\lambda)$ and $d$ separately. In fact, first attempt we tried to fit by a straight line with a common point, but this way was unfruitful. A linear model $a(\lambda)=m \lambda+n$, for each curve of Figure 7 , is not useful because the derivate ratio and the two-frequency ratio are depending not by the two parameters but only by their ratio, $m / n$, so we have no chance to recover them separately. Another attempt, to fit the curve of Figure 7, in the range of our interest, was tried by the model $\operatorname{Exp}(r \lambda+q)$; from Eq. 4 we obtain $r$ but there is no way to obtain $q$ because the ratio in the two different wavelength eliminate it. 
We used therefore the model $\operatorname{Exp}(r \lambda)+q$ to avoid this problem and obtain a linear behavior. In Figure 8 the fit is showed for some different water quality, in the range $420 \mathrm{~nm}$ and $470 \mathrm{~nm}$. Note that in clear water the slope is positive.
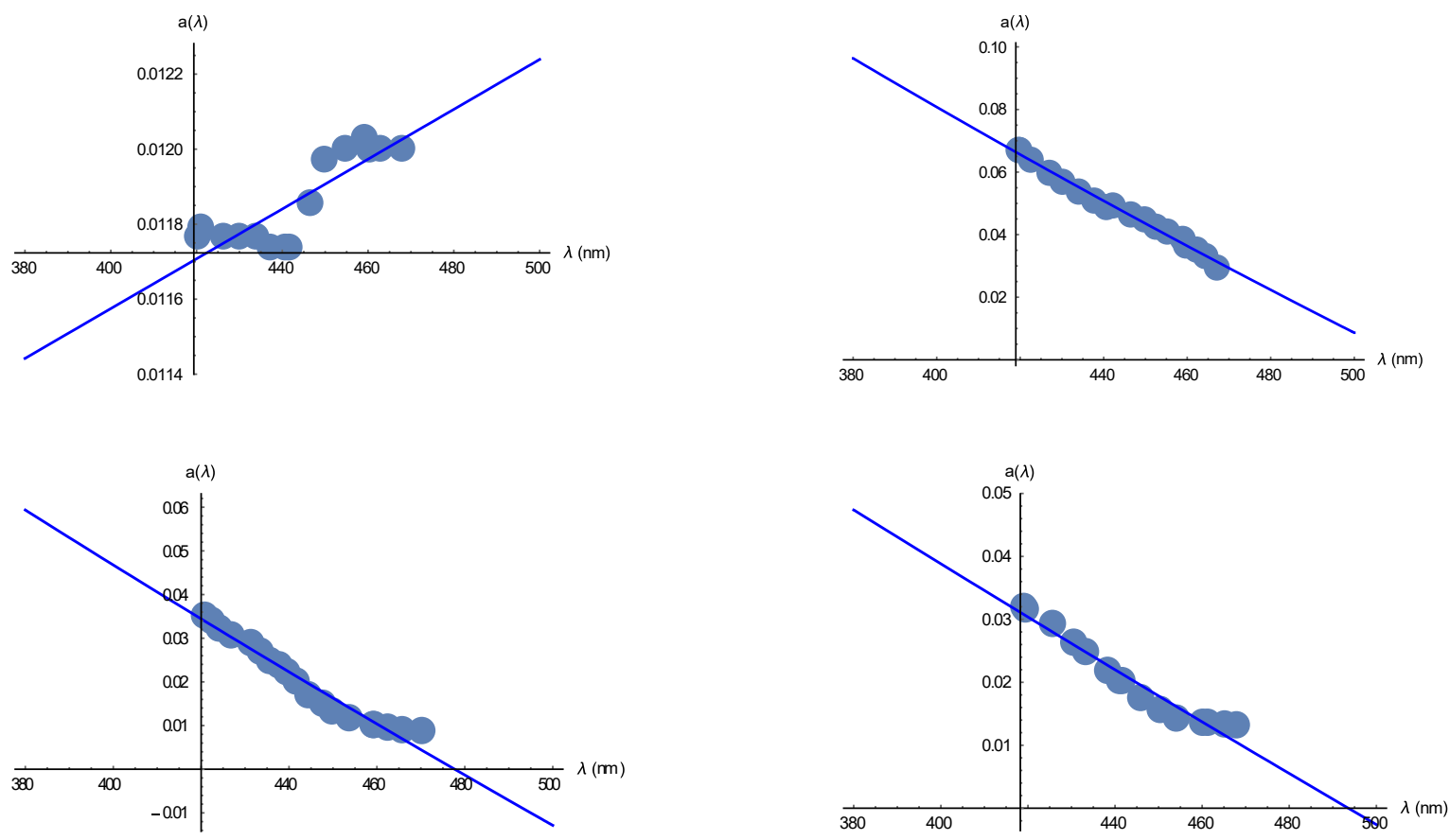

Figure 8 Some examples of fitted coefficient adsorption for different water condition.

We could think to fit the two parameters of the model in the same time using the equations 4 and 5 . This means we have to generate a mixed function to be fitted, taking in account the two equations that are expression of two conditions. Unfortunately, it is well known as the fit would is affected by a huge error, owing to the presence of derivative; we have tried to verified this result and we obtained a good fit for Eq. 4 and Eq. 5 separately but not for the function $a(\lambda)$. As example we have fitted the ratio $a\left(\lambda_{1}\right) / a\left(\lambda_{2}\right)$ and the $a^{\prime}(\lambda) / a(\lambda)$ (See Figure 9 and Figure 10) by combining the two set of data and using a combination of the model functions able to satisfy in the same time all the conditions. From the figures the results are nice, but if you derive the a $(\lambda)$ the result is totally unsatisfactory, as can be seen from Figure 11.

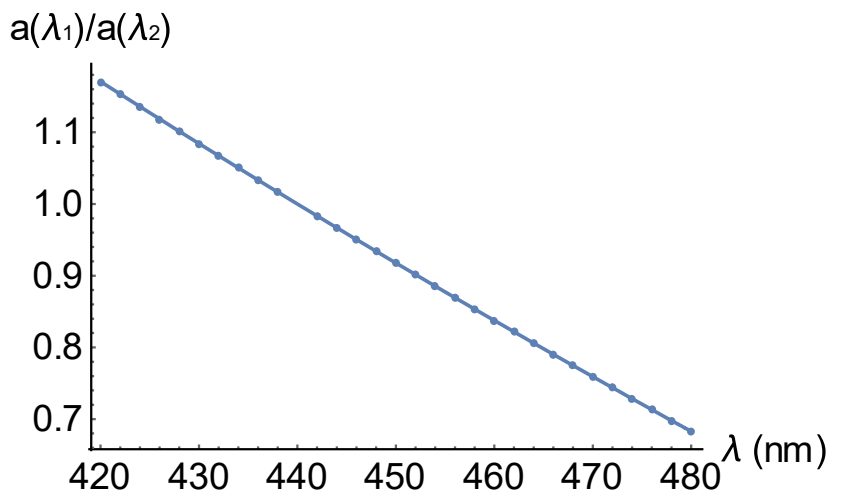

Figure 9 Fit of $a(\lambda 1) / a(\lambda 2)$. 


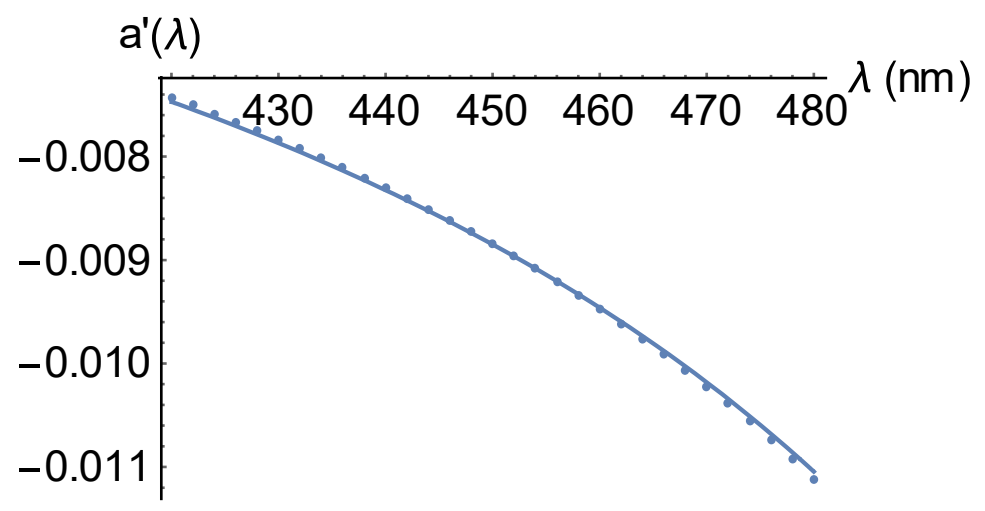

Figure 10 Fit of a' $(\lambda)$

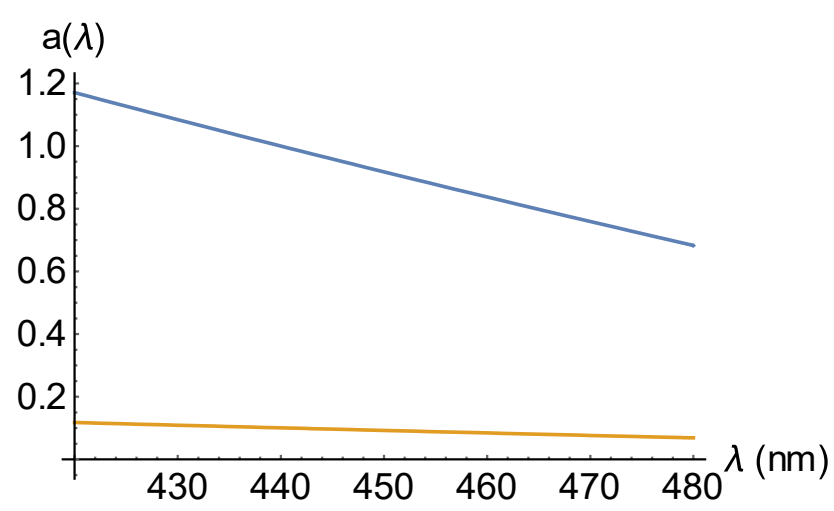

Figure 11 Fit of a $(\lambda)$. The yellow is the real and the blue the unsatisfactory obtained fit.

Note that the results are not depending from the choice of $\lambda_{1}$.

Therefore, we have to fit the two parameters separately. To have success we can get $q$ from Eq. 4 and, consequently, $r$ from Eq. 3.

Many simulations, including the presence of random errors, have been performed obtaining good results. The simulations have been generated starting with known $a\left(\lambda_{i}\right)$ and $d$ and computing the values of $a\left(\lambda_{i}\right) / a\left(\lambda_{1}\right)$ and $a$ ' $\left(\lambda_{i}\right) / a(\lambda)$ for different value of $\lambda_{i}$ and $\lambda_{1}$. We added some random errors to the values. Later, from these values, we fit the vales of $a(\lambda)$ and $d$ founding agreement inside $10 \%$ using all water conditions.

Remember that this system is thought to give an approximation of the absorption coefficient $a(\lambda)$ that can be iteratively improved by successive measurements and/or successive repeated computing.

The case of spherical wave and mobile robots is more realistic and interesting.

We have considered the possibility to move the robots to vary their unknown distance but we still need to vary wave length to obtain $a(\lambda)$. The derivative of Eq. 2 gives as the product $a^{\prime}(\lambda) d$. Computing it for two, unknown, distance $d_{l}$ and $d_{2}$ we can obtain the ratio $d_{l} / d_{2}$ that is what we need. So far, considering the ratio of Eq. 2 for the two distances, we can obtain the product $a(\lambda) d_{l}$. Now, considering the $\log$ of Eq. 2 we have $a(\lambda) d_{l}+\log \left(d_{l}\right)$ and consequently $d_{l}$, without any model of $a(\lambda)$ as done in the preceding case. See at equation (6) and (7).

$\frac{\partial I}{\partial \lambda}=\frac{-a^{\prime}(\lambda) d I_{0} e^{-a(\lambda) d}}{\mathrm{~d}^{2}}$ 


$$
\frac{I\left(d_{1}\right)}{I\left(d_{2}\right)}=\frac{d_{2}^{2} e^{-a(\lambda) d_{1}\left(1-\frac{d_{2}}{d_{1}}\right)}}{d_{1}^{2}}
$$

Experimental measurements into the Bracciano's lake are in progress to verify the real possibility to estimate extinction coefficient $a(\lambda)$ and the distance $d$. Source of errors and imprecision are many. This method must be considered as an iterative method whose precision is increasing with many measurements. These measurements must be integrated with some other source like acoustic, as usual in robot science. The use of more than one LED suddenly increase precision [23]. Working on the ratio $I_{i} / I_{j}$ of the different frequencies we can enhance our measurements because the measured ratio (or a functional form) current by the photodiode remains more stable with respect of that of a single source. The need to have more than one device can be understood intuitively from the wider dynamic available as function the distance at which the signal was collected. Because of the absorption mechanism and on the degradation of the water transparency the choice of the frequencies that can give the maximum sensitivity can change. We are currently selecting sources and classifying standard water qualities, to determine the most suitable functional (i.e. the mentioned intensity ratio) and the approach LED as to the maximum sensitivity of the system. By this way we can be much less sensitive from the variation of the function $a(\lambda)$ and to get an indication of the water pollution degree by comparing the values of the single wavelengths with their ratio.

\section{Conclusion}

This work was written in the frame of cheap underwater swarm robots we are developing in our laboratory. In a swarm the knowledge of its internal configuration is fundamental both for communication between the elements and to adapt its geometry configuration to the mission task. In a preceding paper we have shown as obtain the configuration using the knowledge of the distances between the elements; this is known as a geometry distance problem. In this paper we have presented as a set of commercial cheap LED diodes could be a valid aid, to an acoustic device, to measure distances between them. Modifying power tension, we are able to influence LED's emission peak. The stress of the LED emission peak gives as the opportunity to measure the local, unknown, light adsorption function of the water and, consequently, the distance between the robots. This allow us to avoid the use of expensive variable frequency LED. This research is a collateral effect due to the development of a hybrid acoustic/optical modem, based on the use of cheap commercial LEDs, we are carrying on. These measurements can be used to build the swarm configuration and the relative velocities between its elements. We could use more than one LED with different wavelength; this allow us to reduce experimental errors and increasing the sensitivity of the signal in distance measurements, by enlarging the responsivity dynamic. The use of two or three LEDs of different color enhances dinamic of the measurements increasing the precision of the results. Calculations of the distances in a simulated way show that the error on estimated distance can be significantly reduced.

Many questions are still open like a better management of the LED's emission to render a single light source similar to a multi-source in a controlled way. The work is in progress in our laboratory with experimental campaign into the Bracciano's lake, close to our laboratories.

\section{References}

[1] R. Dell'Erba and C. Moriconi, Bio-inspired Robotics [Online]. Available at: http://www.enea.it/it/produzione-scientifica/edizioni-enea/2014/bio-inspirede-robotics-proceedings.

[2] R. dell'Erba and C. Moriconi, Harness: A robotic swarm for environmental surveillance, in 6th IARP Workshop on Risky Interventions and Environmental Surveillance (RISE), Warsaw, Poland, 2012.

[3] C. Moriconi and R. dell'Erba, The Localization Problem for Harness: A Multipurpose Robotic Swarm, in SENSORCOMM 2012, The Sixth International Conference on Sensor Technologies and Applications, 2012, pagg. 327-333 [Online]. Available at: http://www.thinkmind.org/index.php?view=article\&articleid=sensorcomm_2012_14_20_10138. 
[4] J. J. Leonard, A. A. Bennett, C. M. Smith, and H. Feder, Autonomous underwater vehicle navigation, in IEEE ICRA Workshop on Navigation of Outdoor Autonomous Vehicles, 1998.

[5] B. Anderson e J. Crowell, Workhorse AUV-a cost-sensible new autonomous underwater vehicle for surveys/soundings, search \& rescue, and research, Proc. MTSIEEE OCEANS 2005, pagg. 12281233, 2005.

[6] M. A. Joordens, Design of a low cost underwater robotic research platform, in IEEE International Conference on System of Systems Engineering, 2008. SoSE'08, 2008, pagg. 1-6.

[7] V. Kopman, N. Cavaliere, and M. Porfiri, MASUV-1: A miniature underwater vehicle with multidirectional thrust vectoring for safe animal interactions, IEEEASME Trans. Mechatron., vol. 17, n. 3, pagg. 563-571, 2012.

[8] V. P. Shah, Design considerations for engineering Autonomous Underwater Vehicles (2007). Design considerations for engineering AUVs. [Online]. Available at: http://hdl.handle.net/1721.1/39893

[9] C. Xian-yi, L. Shu-qin, and X. De-shen, Study of self-organization model of multiple mobile robot, Arxiv Prepr. Cs0601062, vol. International Journal of Advanced Robotic Systems, Volume 2, Number 3 (2005), ISSN 1729-8806, 2006. https://doi.org/10.5772/5785

[10] D. Karaboga, An idea based on honey bee swarm for numerical optimization, Technical report-tr06, Erciyes university, engineering faculty, computer engineering department, 2005 [Online]. Available at: http://www-lia.deis.unibo.it/Courses/SistInt/articoli/bee-colony1.pdf.

[11] J. Wiech, V. A. Eremeyev, and I. Giorgio, Virtual spring damper method for nonholonomic robotic swarm self-organization and leader following, Contin. Mech. Thermodyn., vol. 30, n. 5, pagg. 10911102, set. 2018.

[12] A. Battista, P. D’Avanzo, M. Laudato, Discrete systems with geometry-driven evolution: application to 1D elasticity and granular media., Mech. Res. Commun., vol. 92, n. Master Degree Thesis in Physics, Università di Napoli Federico II 2015, pagg. 107-110, 2018.

[13] A. C. Rapisarda, A. Della Corte, R. Drobnicki, F. Di Cosmo, and L. Rosa, A model for bone mechanics and remodeling including cell populations dynamics, Z. Für Angew. Math. Phys., vol. 70, n. 1, pag. 9, nov. 2018.

[14] J. Bachrach, J. Beal, and J. McLurkin, Composable continuous-space programs for robotic swarms, Neural Comput. Appl., vol. 19, n. 6, pagg. 825-847, mag. 2010.

[15] Liu Bo, Swarm Dynamics of a Group of Mobile Autonomous Agents, Chin Pys Lett. 2005, Vol. 22 Issue (1): 254-257

[16] S. Chiesa, S. Taraglio, S. Pagnottelli, and P. Valigi, Flocking approach to spatial configuration control in underwater swarms, 2012, vol. 1, pagg. 313-316 [Online]. Available at: http://www.scopus.com/inward/record.url?eid=2-s2.084867697189\&partnerID=40\&md5=6ba4fec66e104d57f1563f4998bce8cf

[17] R. dell'Erba, «Determination of spatial configuration of an underwater swarm with minimum data», Int. J. Adv. Robot. Syst., vol. 12, n. 7, pag. 97, 2015.

[18] F. Di Cosmo, M. Laudato, and M. Spagnuolo, Acoustic Metamaterials Based on Local Resonances: Homogenization, Optimization and Applications, in Advanced Structured Materials, 2018, pagg. 247-274.

[19] E. Barchiesi, M. Spagnuolo, and L. Placidi, Mechanical metamaterials: a state of the art, Math. Mech. Solids, vol. 24, n. 1, pagg. 212-234, feb. 2018.

[20] E. Turco, Tools for the numerical solution of inverse problems in structural mechanics: review and research perspectives, Eur. J. Environ. Civ. Eng., vol. 21, n. 5, pagg. 509-554, mag. 2017.

[21] I. Giorgio, N. L. Rizzi, and E. Turco, Continuum modelling of pantographic sheets for out-of-plane bifurcation and vibrational analysis, Proc. R. Soc. Math. Phys. Eng. Sci., vol. 473, n. 2207, pag. 20170636, nov. 2017.

[22] J.-J. Alibert, P. Seppecher, and F. Dell'isola, Truss modular beams with deformation energy depending on higher displacement gradients, Math. Mech. Solids, vol. 8, n. 1, pagg. 51-73, 2003. 
[23] R. dell'Erba and C. Moriconi, High power leds in localization of underwater robotics swarms, IFAC-Pap., vol. 48, n. 10, pagg. 117-122, 2015.

[24] Mark Rhodes, Electromagnetic Propagation through the Water Column, 2004. Available at: www.wirelessfibre.co.uk

[25] M. Dunbabin, I. Vasilescu, P. Corke, and D. Rus, Experiments with Cooperative Networked Control of Underwater Robots, in Experimental Robotics, 2008, pagg. 463-470.

[26] W. Bogdan and D. Jerzi, Light Absorption in Sea Water, Springer. 2007 [Online]. Available at: http://download.springer.com/static/pdf/107/bok\%253A978-0-387-495606.pdf?auth66=1416993589_ea5bcc47d8e3ca441bbb50259745bb4b\&ext=.pdf.

[27] G. Rosi, I. Giorgio, and V. A. Eremeyev, Propagation of linear compression waves through plane interfacial layers and mass adsorption in second gradient fluids, ZAMM-Journal Appl. Math. Mech. Für Angew. Math. Mech., vol. 93, n. 12, pagg. 914-927, 2013.

[28] G. Rosi, L. Placidi, and F. dell'Isola, "Fast" and "slow" pressure waves electrically induced by nonlinear coupling in Biot-type porous medium saturated by a nematic liquid crystal, Z. Für Angew. Math. Phys., vol. 68, n. 2, pag. 51, mar. 2017.

[29] V. A. Eremeyev, G. Rosi, and S. Naili, Comparison of anti-plane surface waves in strain-gradient materials and materials with surface stresses, Math. Mech. Solids, vol. 24, n. 8, pagg. 2526-2535, mag. 2018.

[30] F. Dell'Isola, A. Madeo, and L. Placidi, Linear plane wave propagation and normal transmission and reflection at discontinuity surfaces in second gradient 3D continua, ZAMM Z. Angew. Math. Mech., vol. 92, n. 1, pagg. 52-71, 2011.

[31] L. Placidi, G. Rosi, I. Giorgio, and A. Madeo, Reflection and transmission of plane waves at surfaces carrying material properties and embedded in second-gradient materials, Math. Mech. Solids, vol. 19, n. 5, pagg. 555-578, mar. 2013.

[32] I. A. Abbas, A.-E.-N. N. Abdalla, F. S. Alzahrani, and M. Spagnuolo, Wave propagation in a generalized thermoelastic plate using eigenvalue approach, J. Therm. Stress., vol. 39, n. 11, pagg. 1367-1377, nov. 2016.

[33] E. Barchiesi, M. Laudato, and F. Cosmo, Wave dispersion in non-linear pantographic beams, Mech. Res. Commun., vol. 94, nov. 2018. 